\title{
Mean Platelet Volume and Mean Platelet Volume/Platelet Count Ratio as Diagnostic Markers for Hepatocellular Carcinoma in Chronic Hepatitis C Patients
}

\author{
Maha Z. Omar ${ }^{1}$, Mohebat H. Gouda ${ }^{2}$, Mona M. Elbehisy ${ }^{3}$ \\ ${ }^{1}$ Department of Hepatology, Gastroenterology and Infectious Diseases, faculty of Medicine, Benha \\ University, Egypt \\ ${ }^{2}$ Department of Pathology, faculty of Medicine, Benha University, Egypt \\ ${ }^{3}$ Department of Clinical Pathology, faculty of Medicine, Benha University, Egypt
}

Corresponding Author

Maha Z. Omar

Mobile: +

01223287312

E mail: mahazeinelabedin@ya $\underline{\text { hoo.com }}$

Key words: $M P V, M P V / P C, A F P$, HCC
Background and study aim: Hepatocellular carcinoma (HCC) is the most common primary malignant tumor of the liver. AFP is the gold standard tumor marker for HCC, mean platelet volume (MPV) is a parameter obtained from complete blood count (CBC) by automated analyzers, shown to be increased in multiple malignancies and inflammatory conditions. This prospective study was designed to evaluate the diagnostic usefulness of MPV and MPV/ platelet count (PC) ratio in HCC patients due to chronic hepatitis $\mathrm{C}$ (CHC) infection.

Materials and Methods: One hundred and twenty subjects enrolled in this study, they were divided into 4 equal groups: group I included 30 healthy subjects (control group), group II included 30 patients with chronic viral hepatitis without cirrhosis, group III included 30 cirrhotic patients without HCC and group IV included 30 cirrhotic patients with HCC.MPV, $\mathrm{MPV} / \mathrm{PC}$ ratio \& AFP were evaluated in all groups. Triphasic CT was done for patients of group IV to confirm the diagnosis of HCC. Liver biopsy was done for patients of group II to confirm the diagnosis of chronic hepatitis $\mathrm{C}$.

Results: MPV showed higher levels in HCC group compared to control, CHC, and cirrhotic groups with $\mathrm{p}$ value $(<0.001)$ but there was no statistically significant difference between HCC group and

\section{INTRODUCTION}

Hepatocellular carcinoma (HCC) is the second leading cause of cancer deaths in the world with more than 745,000 new deaths annually [1]. The prognosis of $\mathrm{HCC}$ remains poor, and cirrhotic group ( $\mathrm{p}$ value $=0.49$ ), while $\mathrm{MPV} /$ platelet count ratio was higher in cirrhotic group $\left(0.19 \pm 0.09 \mathrm{fL} / 10^{9} / \mathrm{L}\right)$ than HCC group $\left(0.14 \pm 0.08 \mathrm{fL} / 10^{9} / \mathrm{L}\right)$ but also with no significant differences between both groups $(p=0.06)$. There was insignificant positive correlation between MPV and AFP in HCC group $(\rho=0.11$ and $p$ value $=0.57$ ). Also, there was insignificant negative correlation between MPV/PC and AFP in HCC group $(\rho=-0.17$ and $p$ value $=0.37)$. In receiver operating characteristic (ROC) curve analysis, MPV had high sensitivity (73.33\%), specificity (70 \%), and area under curve (AUC) was 0.7, So it more better than MPV/platelet count ratio in diagnosis of $\mathrm{HCC}$ which had sensitivity $(76.67 \%)$, low specificity $(56.67 \%)$, and AUC was 0.63 , while AFP had much higher sensitivity (90\%), specificity $(98.33 \%)$ than both studied parameters (MPV, MPV/PC ratio) with highly statistically difference when compared to MPV $(\mathrm{p}<0.001)$ and area under curve (AUC) was 0.9.

Conclusion: MPV and MPV/PC ratio are less sensitive and specific than AFP in diagnosis of HCC. So AFP is still the gold standard marker in diagnosis of HCC and MPV and MPV/PC ratio may be used only in association with other markers like AFP to improve sensitivity of tumor detection.

most patients have a 5-years survival rate of less than $5 \%$ mainly because of the late diagnosis [2]. Although it is obvious that development of new diagnostic modalities will significantly increase the detection rate of $\mathrm{HCC}$, 
there is still a need for detection methods. AFP is the most established tumor marker in HCC and the gold standard by which other markers for the disease are judged [3]. Mean platelet volume (MPV) is a parameter of routine blood count which was actively investigated in many liver diseases. MPV was found to be related to metabolic syndrome [4], advanced liver fibrosis [5]. And ascitic fluid infection [6]. There is an inverse relationship between platelet size and number. When platelets decrease in number, bone marrow megakaryocytes are stimulated by thrombopoietin and their nucleus becomes hyperlobulated, with much higher DNA content $[\mathbf{7 , 8}]$ producing larger platelets. Thus, platelets with a higher MPV are expected to be seen in destructive thrombocytopenia when megakaryocytic stimulation is present $[\mathbf{9 , 1 0}]$.

This study was aimed to evaluate the diagnostic usefulness of MPV and MPV/PC ratio in HCC patients due to chronic hepatitis $\mathrm{C}$ infection.

\section{MATERIALS AND METHODS}

This prospective study was conducted on 120 subjects admitted to Hepatology , Gastroenterology and Infectious Diseases Department ,Benha University Hospitals with mean age $45.29 \pm 16.44$ years in period from April 2014 to March 2016.

The subjects were divided into 4 groups :

Group I: included 30 healthy subjects served as control group.

Group II: included 30 patients with chronic hepatitis $\mathrm{C}$.

Group III: included 30 cirrhotic patients without HCC.

Group IV: included 30 cirrhotic patients with HCC.

All studied patients were given an informed written concent for participation in this study, the protocol of this study was approved from ethical committe of Benha faculty of medicine, Benha university.

Patients with hepatic malignancy rather than HCC, any signs of inflammation, acute myocardial ischemia, atherosclerosis and cerebrovascular events, ulcerative colitis, Crohn's disease, rheumatoid disease, portal vein thrombosis and patients receiving drugs affecting platelet count or function as Aspirin were excluded from study.
- Diagnosis of CHC infection was done by following criteria:

1- Detectable HCV Abfor more than 6 months.

2- Positivity of HCV RNA and confirmed by liver biopsy.

- Diagnosis of liver cirrhosis was based on laboratory investigations and radiological findings.

- Diagnosis of HCC was based on serum level of AFP and imaging modalities( pelvi-abdominal $\mathrm{U} / \mathrm{S}$ and triphasic $\mathrm{CT}$ abdomen)

- All subjects were subjected to full history taking and thorough clinical examination and laboratory investigations including MPV, MPV/PC ratio and AFP, triphasic CT was done to confirm HCC cases.

\section{Statistical analysis}

Statistical analysis was performed using PASW statistics 18 (SPSS, Chicago, IL USA). Statistically significant differences were analyzed by the X2 test for categorical variables. Continuous variables were tested for normality by the KolmogorovSmirnov test. Normally distributed data are presented as mean and standard deviations (SDs). The sensitivity and specificity of MPV level. MPV/PC ratio and AFP for a diagnosis of hepatocellular carcinoma was done under various cut off ranges and receiver operating characteristics (ROC) curves were drawn. Pearson and Spearman correlation between AFP and MPV, MPV/PC ratio in HCC group were calculated. A two tailed $\mathrm{P}$ value below 0.05 was considered statistically significant.

\section{RESULTS}

There was highly statistically significant difference between studied groups as regard age and sex. $(\mathrm{p}<0.001)$.There was male predominance in HCC group with male to female ratio $4: 1$. The mean age was higher in cirrhotic group without HCC than HCC group with no statistically significant difference between HCC and cirrhotic groups. $(\mathrm{p}=0.52)$, while there was no statistically significant difference between studied groups as regard diabetes mellitus and systemic hypertension as showed in table (1). Highly statistically significant difference was found between studied groups (I, II, III and IV) as regard all studied laboratory parameters, AFP was highly elevated in HCC group than in cirrhotic, chronic hepatitis and control groups with highly statistically 
significant difference $(\mathrm{P}<0.001)(1239.93 \pm 2881.97$ $\mathrm{ng} / \mathrm{dl} \mathrm{Vs} 18.35 \pm 17.0 \mathrm{ng} / \mathrm{dl}$ and $2.2 \pm 1.14 \mathrm{ng} / \mathrm{dl}$ Vs $0.34 \pm 0.31 \mathrm{ng} / \mathrm{dl}$ ) respectively (Table 2 ).

MPV and MPV/platelet count ratio show highly statistically significant difference between studied groups (I,II,III and IV) $(\mathrm{P}<0.001)$. HCC group shows higher levels of MPV compared to other groups $(10.94 \pm 1.88 \mathrm{fL}$ in HCC group Vs $10.74 \pm$ $1.21 \mathrm{fL}$ in cirrhotic group , 9.13 $\pm 1.74 \mathrm{fL}$ in $\mathrm{HCV}$ group and $8.07 \pm 0.86 \mathrm{fL}$ in control group) with $\mathrm{p}$ value $<0.001$, but there was no statistically significant difference between HCC group and cirrhotic group ( $\mathrm{p}$ value=0.49). While MPV/ platelet count ratio was higher in cirrhotic group $\left(0.19 \pm 0.09 \mathrm{fL} / 10^{9} / \mathrm{L}\right)$ than HCC group $(0.14 \pm$ $\left.0.08 \mathrm{fL} / 10^{9} / \mathrm{L}\right)$, chronic hepatitis group $(0.05 \pm 0.02$ $\left.\mathrm{fL} / 10^{9} / \mathrm{L}\right)$ and control group $\left(0.03 \pm 0.01 \mathrm{fL} / 10^{9} / \mathrm{L}\right)$. There was no significant differences between cirrhotic group and HCC group $(\mathrm{p}=0.06)$. Table (3) and figure $(1,2)$.There was insignificant positive correlation between MPV and AFP $(\rho=0.11$ and $\mathrm{p}$ value $=0.57$ ), and insignificant negative correlation between MPV/ PC and AFP in HCC group ( $\rho=-$ 0.17 and $p$ value $=0.37$ ) (Table 4). At cut off level $>10.7 \mathrm{fl}$, MPV had high sensitivity (73.33\%), specificity $(70 \%), \mathrm{NPV}=(83 \%)$, low $\mathrm{PPV}=$ $(51.2 \%)$, accuracy $(71.11 \%)$ and area under curve (AUC) was 0.7, So it more better than MPV/platelet count ratio in diagnosis of HCC which had at cut off level $>0.11 \mathrm{fl} / 10^{9} / \mathrm{L}$, low specificity $(56.67 \%)$ and area under curve (AUC) was 0.63 with no statistically significant difference when compared to MPV ( $\mathrm{p}=0.14$ ). While AFP at cut off $>62.3$ ng/dl level had much higher sensitivity (90\%), specificity $(98.33 \%), \mathrm{PPV}=(96.43 \%), \mathrm{NPV}=$ (95.16\%), accuracy $(95.56 \%)$ and area under curve (AUC) was 0.9 , with highly statistically significant difference when compared to MPV $(\mathrm{p}=<0.001)$. Table (5) and Figure (3). So, AFP is still better than other studied parameters in diagnosis of HCC.

Table (1): Comparison between studied groups as regard baseline data.

\begin{tabular}{|c|c|c|c|c|c|c|c|c|c|c|c|}
\hline \multirow{2}{*}{\multicolumn{2}{|c|}{ Variables }} & \multicolumn{2}{|c|}{$\begin{array}{c}\text { Group I } \\
\text { (control } \\
\text { group) } \\
(\text { No. }=30)\end{array}$} & \multicolumn{2}{|c|}{$\begin{array}{c}\text { Group II } \\
\text { (HCV } \\
\text { group) } \\
(\text { No.=30) }\end{array}$} & \multicolumn{2}{|c|}{$\begin{array}{c}\text { Group III } \\
\text { (cirrhosis } \\
\text { without HCC) } \\
(\text { No.=30) }\end{array}$} & \multicolumn{2}{|c|}{$\begin{array}{c}\text { Group IV } \\
\text { (HCC } \\
\text { group) } \\
(\text { No.=30) } \\
\end{array}$} & \multirow[t]{2}{*}{ Test } & \multirow[t]{2}{*}{$\mathbf{P}$} \\
\hline & & No & $\%$ & No. & $\%$ & No. & $\%$ & No. & $\%$ & & \\
\hline \multirow{2}{*}{ Sex } & Females & 24 & 80.0 & 15 & 50.0 & 18 & 60.0 & 6 & 20.0 & \multirow{2}{*}{$\begin{array}{c}\chi^{2}= \\
22.56\end{array}$} & \multirow[t]{2}{*}{$<0.001 * *$} \\
\hline & Males & 6 & 20.0 & 15 & 50.0 & 12 & 40.0 & 24 & 80.0 & & \\
\hline $\begin{array}{l}\text { Age } \\
\text { (years) }\end{array}$ & $\begin{array}{l}\text { Mean } \\
\pm \mathrm{SD} ; \\
\text { (range) }\end{array}$ & \multicolumn{2}{|c|}{$\begin{array}{c}27.37 \pm 9.78 \\
\quad(16-48)\end{array}$} & \multicolumn{2}{|c|}{$\begin{array}{c}\dagger 37.27 \pm 10.3 \\
2 ;(20-58)\end{array}$} & \multicolumn{2}{|c|}{$\begin{array}{c}\dagger+59 \pm 9.54 \\
(34-79)\end{array}$} & \multicolumn{2}{|c|}{$\begin{array}{c}\dagger+57.53 \pm 8.1 \\
8 ;(40-74)\end{array}$} & $\mathrm{F}=80.32$ & $<0.001 * *$ \\
\hline \multirow{2}{*}{ DM } & No & 27 & 90.0 & 24 & 80.0 & 23 & 76.67 & 20 & 66.67 & \multirow{2}{*}{$\chi^{2}=4.91$} & \multirow{2}{*}{0.18} \\
\hline & Yes & 3 & 10.0 & 6 & 20.0 & 7 & 23.33 & 10 & 33.33 & & \\
\hline \multirow{2}{*}{ HTN } & No & 27 & 90.0 & 26 & 86.67 & 28 & 93.33 & 25 & 83.33 & \multirow{2}{*}{ FET } & \multirow{2}{*}{0.78} \\
\hline & Yes & 3 & 10.0 & 4 & 13.33 & 2 & 6.67 & 5 & 16.67 & & \\
\hline
\end{tabular}

* Significant $(\mathrm{P}<0.05)$

** Highly Significant $(\mathrm{P}<0.001)$

$\dagger$ Significant differences compared to group I

+ Significant differences compared to Group II

- (HCC group against cirrhosis group as regard age showed $\mathrm{p}=0.52$ ) 
Table (2) Comparison between studied groups (I,II, III \&IV) regarding laboratory findings.

\begin{tabular}{|c|c|c|c|c|c|c|}
\hline \multirow[t]{2}{*}{ Variables } & $\begin{array}{c}\text { Group I } \\
\text { (control } \\
\text { group) } \\
\text { (No.=30) }\end{array}$ & $\begin{array}{c}\text { Group II } \\
\text { (HCV } \\
\text { group) } \\
\text { (No.=30) }\end{array}$ & $\begin{array}{c}\text { Group III } \\
\text { (cirrhosis } \\
\text { without } \\
\text { HCC) } \\
(\text { No.=30 }\end{array}$ & $\begin{array}{c}\text { Group IV } \\
\text { (HCC } \\
\text { group) } \\
\text { (No.=30) }\end{array}$ & \multirow[t]{2}{*}{ Test } & \multirow[t]{2}{*}{$\mathbf{P}$} \\
\hline & \multicolumn{4}{|c|}{ Mean \pm SD } & & \\
\hline FBS (mg/dl) & $\begin{array}{l}94.1 \pm \\
25.85\end{array}$ & $\begin{array}{c}99.17 \pm \\
25.69\end{array}$ & $\begin{array}{c}! \dagger 131.97 \pm \\
60.14\end{array}$ & $\begin{array}{c}! 120.73 \pm \\
60.01\end{array}$ & $\chi^{2}=16.48$ & $<0.001 * *$ \\
\hline HB (gm/dl) & $\begin{array}{c}12.4 \pm \\
1.27\end{array}$ & $\begin{array}{c}13.47 \pm \\
1.41\end{array}$ & $\begin{array}{c}! \dagger 10.15 \pm \\
1.61\end{array}$ & $\begin{array}{c}\dagger+11.28 \pm \\
2.25\end{array}$ & $\mathrm{~F}=21.85$ & $<0.001 * *$ \\
\hline WBCs $\left(10^{3} / \mathrm{cmm}\right)$ & $\begin{array}{c}6.14 \pm \\
1.68\end{array}$ & $\begin{array}{c}5.81 \pm \\
1.69\end{array}$ & $\begin{array}{l}! 4.85 \pm \\
2.78\end{array}$ & $\begin{array}{c}5.85 \pm \\
3.04\end{array}$ & $\chi^{2}=10.24$ & $0.02 *$ \\
\hline Platelets $\left(10^{9} / \mathrm{L}\right)$ & $\begin{array}{c}247.77 \pm \\
61.03\end{array}$ & $\begin{array}{c}210.1 \pm \\
74.98\end{array}$ & $\begin{array}{c}1 \dagger 67.07 \pm \\
26.53 \\
\end{array}$ & $\begin{array}{c}! \dagger 103.1 \pm \\
65.24\end{array}$ & $F=61.65$ & $<0.001 * *$ \\
\hline $\begin{array}{l}\text { S. creatinine } \\
(\mathrm{mg} / \mathrm{dl})\end{array}$ & $\begin{array}{c}0.79 \pm \\
0.14\end{array}$ & $\begin{array}{c}0.86 \pm \\
0.20\end{array}$ & $\begin{array}{l}! 1.16 \pm \\
0.64\end{array}$ & $\begin{array}{c}! \dagger 1.14 \pm \\
0.59\end{array}$ & $\chi^{2}=24.35$ & $<0.001 * *$ \\
\hline ALT (IU) & $\begin{array}{c}23.77 \pm \\
6.5\end{array}$ & $\begin{array}{l}50.2 \pm \\
29.47\end{array}$ & $\begin{array}{l}149.03 \pm \\
44.15\end{array}$ & $\begin{array}{l}50.31 \pm \\
28.14\end{array}$ & $\chi^{2}=37.41$ & $<0.001 * *$ \\
\hline AST (IU) & $\begin{array}{c}24.07 \pm \\
6.9\end{array}$ & $\begin{array}{l}! 43.18 \pm \\
24.45\end{array}$ & $\begin{array}{l}! 57.2 \pm \\
34.11\end{array}$ & $\begin{array}{l}! 60.03 \pm \\
30.99\end{array}$ & $\chi^{2}=48.17$ & $<0.001 * *$ \\
\hline $\begin{array}{ll}\text { T. } & \text { bilirubin } \\
(\mathrm{mg} / \mathrm{dl}) & \end{array}$ & $\begin{array}{c}0.52 \pm \\
0.45\end{array}$ & $\begin{array}{c}0.72 \pm \\
0.27\end{array}$ & $\begin{array}{c}! \nmid 3.2 \pm \\
1.93\end{array}$ & $\begin{array}{c}! \dagger 3.14 \pm \\
3.37\end{array}$ & $\chi^{2}=71.36$ & $<0.001 * *$ \\
\hline $\begin{array}{ll}\text { S. } & \text { albumin } \\
(\mathrm{gm} / \mathrm{dl}) & \\
\end{array}$ & $\begin{array}{c}4.6 \pm \\
0.37 \\
\end{array}$ & $\begin{array}{c}! 4.24 \pm \\
0.71\end{array}$ & $\begin{array}{c}! \dagger 2.52 \pm \\
0.39\end{array}$ & $\begin{array}{c}\dagger 2.78 \pm \\
0.61\end{array}$ & $\mathrm{~F}=111.24$ & $<0.001 * *$ \\
\hline INR & $\begin{array}{c}1.01 \pm \\
0.05\end{array}$ & $\begin{array}{c}! 1.28 \pm \\
0.18\end{array}$ & $\begin{array}{c}11.46 \pm \\
0.34\end{array}$ & $\begin{array}{l}! \dagger 1.55 \pm \\
0.44\end{array}$ & $\mathrm{~F}=19.07$ & $<0.001 * *$ \\
\hline PT(sec) & $\begin{array}{c}12.15 \pm \\
0.49\end{array}$ & $\begin{array}{c}! 13.79 \pm \\
1.23\end{array}$ & $\begin{array}{c}! \dagger 16.25 \pm \\
2.57 \\
\end{array}$ & $\begin{array}{c}! \dagger 16.74 \pm \\
3.33 \\
\end{array}$ & $\mathrm{~F}=28.56$ & $<0.001 * *$ \\
\hline $\begin{array}{l}\text { PCR HCV RNA } \\
(\text { IU/ml) }\end{array}$ & - & $\begin{array}{l}425981.3 \pm \\
386821.5\end{array}$ & - & - & - & - \\
\hline AFP(ng/dl) & $\begin{array}{c}0.34 \pm \\
0.31\end{array}$ & $\begin{array}{c}12.2 \pm \\
1.14\end{array}$ & $\begin{array}{c}! 18.35 \pm \\
17.0\end{array}$ & $\begin{array}{l}! \dagger+1239.93 \\
\pm 2881.97\end{array}$ & $\begin{array}{c}\chi^{2}= \\
103.36\end{array}$ & $<0.001 * *$ \\
\hline
\end{tabular}

! Significant differences compared to Group I

$\dagger$ Significant differences compared to group II

† Significant differences compared to Group III

Table (3): Comparison between studied groups (I, II, III \&IV) regarding MPV\& MPV/Platelet count ratio.

\begin{tabular}{|c|c|c|c|c|c|c|}
\hline \multirow[t]{2}{*}{ Variables } & $\begin{array}{c}\text { Group I } \\
\text { (control } \\
\text { group) } \\
\text { (No.=30) } \\
\end{array}$ & $\begin{array}{l}\text { Group II } \\
\text { (HCV } \\
\text { group) } \\
\text { (No.=30) } \\
\end{array}$ & $\begin{array}{c}\text { Group III } \\
\text { (cirrhosis } \\
\text { without HCC) } \\
(\text { No.=30) } \\
\end{array}$ & $\begin{array}{c}\text { Group IV } \\
\text { (HCC } \\
\text { group) } \\
\text { (No.=30) } \\
\end{array}$ & \multirow[t]{2}{*}{ Test } & \multirow[t]{2}{*}{$\mathbf{P}$} \\
\hline & Mean \pm SD & Mean \pm SD & Mean \pm SD & Mean \pm SD & & \\
\hline $\begin{array}{l}\text { MPV(fL) } \\
\text { Normal range } \\
(8.9 \pm 1.4 \mathrm{Fl})\end{array}$ & $\begin{array}{c}8.07 \pm \\
0.86\end{array}$ & $\begin{array}{c}! 9.13 \pm \\
1.74\end{array}$ & $\begin{array}{c}! \dagger 10.74 \pm \\
1.21\end{array}$ & $\begin{array}{c}! \uparrow .10 .94 \pm \\
1.08\end{array}$ & $\mathrm{~F}=34.95$ & $<0.001 * *$ \\
\hline $\begin{array}{l}\text { MPV/Platelet } \\
\text { count }(\text { fL } / 109 / \mathrm{L})\end{array}$ & $\begin{array}{c}0.03 \pm \\
0.01\end{array}$ & $\begin{array}{c}0.05 \pm \\
0.02\end{array}$ & $\begin{array}{c}\dagger 0.19 \pm \\
0.09\end{array}$ & $\begin{array}{c}\dagger+0.14 \pm \\
0.08\end{array}$ & $F=43.69$ & $<0.001 * *$ \\
\hline
\end{tabular}

! Significant differences compared to Group I

$\dagger$ Significant differences compared to group II

- (HCC group against cirrhosis group as regard MPV showed $\mathrm{p}=0.49$ )

- (HCC group against cirrhosis group as regard MPV/PC ratio showed $\mathrm{p}=0.06$ ) 
Table (4): Correlation between AFP and MPV, MPV/PC ratio in HCC group.

\begin{tabular}{|c|c|c|c|c|}
\hline \multirow{2}{*}{ Variables } & \multicolumn{4}{|c|}{ HCC group (N0=30) } \\
\cline { 2 - 5 } & \multicolumn{2}{|c|}{ MPV } & MPV/ PC ratio \\
\cline { 2 - 5 } & Correlation coefficient & $\mathbf{P}$ & Correlation coefficient & P \\
\hline AFP (ng/dl) & $\rho=0.11$ & 0.57 & $\rho=-0.17$ & 0.37 \\
\hline
\end{tabular}

r: Pearson correlation coefficient

$\rho$ : Spearman correlation coefficient

Table (5): Diagnostic performance of MPV, MPV/platelet count ratio and AFP for diagnosis of HCC

\begin{tabular}{|c|c|c|c|c|c|c|c|c|c|}
\hline Variable $s$ & 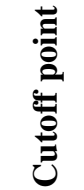 & 氙 & 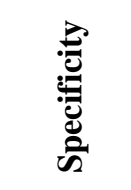 & 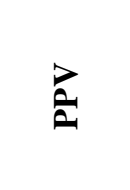 & z & 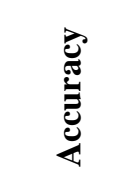 & 己 & $\begin{array}{l}\Xi \\
\text { o̊ } \\
2\end{array}$ & $\mathbf{P}^{*}$ \\
\hline $\begin{array}{l}\text { MPV } \\
\text { (fL) }\end{array}$ & 10.7 & $73.33 \%$ & $70.0 \%$ & $51.2 \%$ & $83.0 \%$ & $71.11 \%$ & 0.7008 & $\begin{array}{c}0.59- \\
0.79\end{array}$ & \\
\hline $\begin{array}{l}\text { MPV/PC } \\
\left(\mathrm{Fl} /{ }_{10}{ }^{9} / \mathrm{L}\right)\end{array}$ & 0.11 & $76.67 \%$ & $56.67 \%$ & $46.9 \%$ & $82.9 \%$ & $63.33 \%$ & 0.6217 & $\begin{array}{c}0.51- \\
0.72\end{array}$ & 0.14 \\
\hline $\begin{array}{l}\text { AFP } \\
\text { (ng/dl) }\end{array}$ & 62.3 & $90.00 \%$ & $98.33 \%$ & $96.43 \%$ & $95.16 \%$ & $95.56 \%$ & 0.9744 & $\begin{array}{c}0.92- \\
1.00\end{array}$ & $<0.001 * *$ \\
\hline
\end{tabular}

*Compared to MPV

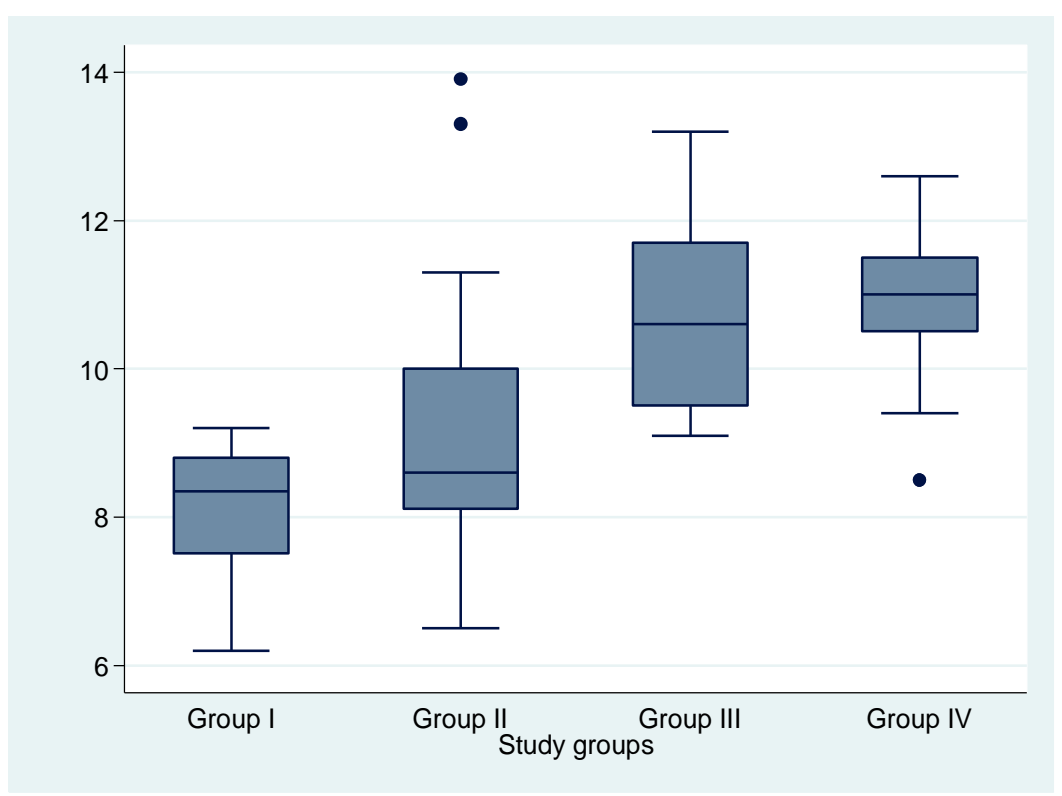

Figure (1): Box plot showing MPV level among studied groups 


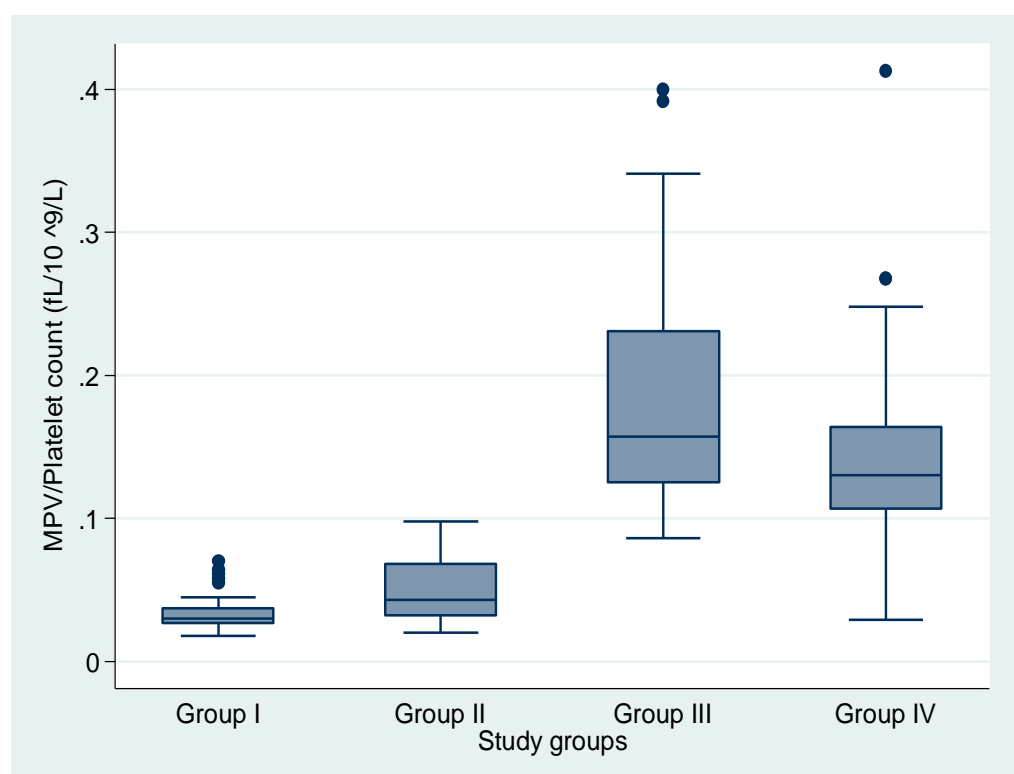

Figure (2): Box plot showing MPV / PC ratio among studied groups

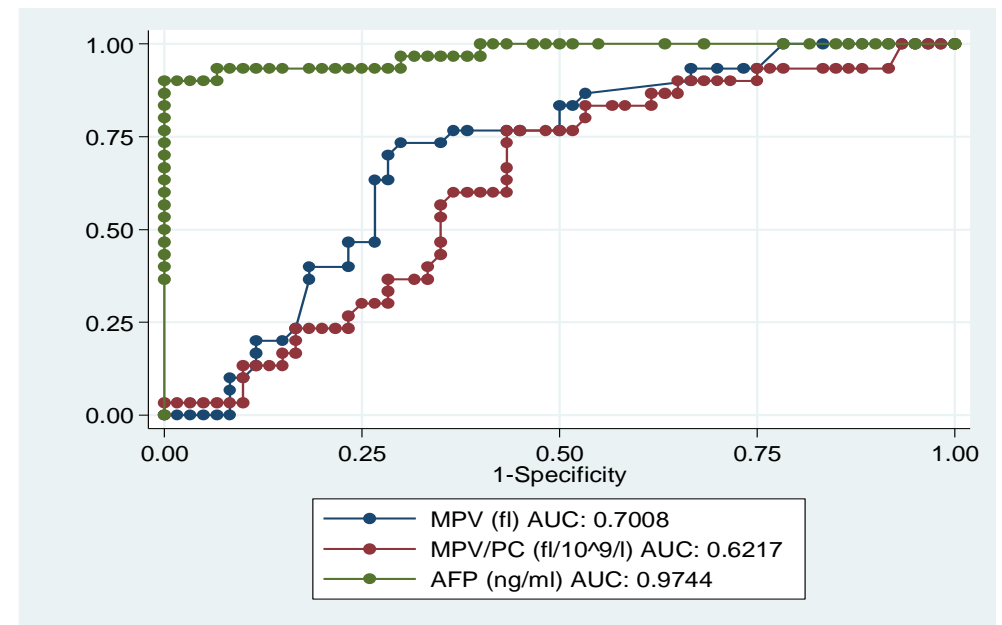

Figure (3): Roc curve for diagnostic performance of MPV, MPV/PC ratio and AFP in diagnosis of HCC

\section{DISCUSSION}

Liver cancer is the second cause of cancer related death worldwide $(745,000$ cases per year) [1], and accounts for $7 \%$ of all cancers, representing more than $90 \%$ of primary liver cancers [11]. Most hepatocellular carcinomas are diagnosed at intermediate or advanced stages and only $30 \%$ of patients benefit from curative therapies such as resection, liver transplantation or percutaneous ablation [12]. This study was aimed to evaluate the diagnostic usefulness of MPV and MPV/PC ratio in $\mathrm{HCC}$ patients due to chronic hepatitis $\mathrm{C}$ infection. There was highly statistically significant difference between studied groups as regard age and sex. $(\mathrm{p}<0.001)$ with male predominance in HCC group (male to female ratio was 4:1), this result was in agreement with previous studies which showed that there was a male predominance among HCC patients with male to female ratio of 3.6:1 [13,14]. In contrast to our results, several studies reported that a non significant difference in sex distribution between HCC patients [15]. The mean age was higher in cirrhotic group without HCC than HCC group with no statistically significant difference between $\mathrm{HCC}$ and cirrhotic groups. $(p=0.52)$, this result was in agreement with previous study which showed that the mean 
age of HCC patients was $57.95 \pm 8.41$ years [14] and in agreement with another study which documented that the mean age was 56.28 years for the HCC patients [16]. Also, another study reported that the most predominant age group for HCC was (40-59) years [17]. In contrast to our results, other studies reported that reported that HCC occur in older age (61 years) [18] and (66 years) [19]. AFP was markedly elevated in HCC group than in cirrhotic, chronic hepatitis and control groups with highly statistically significant difference $(\mathrm{P}<0.001) \quad(1239.93 \pm 2881.97 \mathrm{ng} / \mathrm{dl}$, $18.35 \pm 17.0 \mathrm{ng} / \mathrm{dl}, 2.2 \pm 1.14 \mathrm{ng} / \mathrm{dl}$ and $0.34 \pm 0.31$ $\mathrm{ng} / \mathrm{dl}$ ) respectively, these results were in agreement with a previous study who mentioned that marked elevation of AFP level was observed in patients with $\mathrm{HCC}$ in comparison with healthy control subjects, patients with $\mathrm{CHC}$ and patients LC (239.5 $\pm 770.7 \mathrm{ng} / \mathrm{dl}$ in HCC group, 7.1 $\pm 5.4 \mathrm{ng}$ $/ \mathrm{dl}$ in control group, $10.1 \pm 10.7 \mathrm{ng} / \mathrm{dl}$ in CHC group and $31.1 \pm 83.2 \mathrm{ng} / \mathrm{dl}$ in cirrhotic group). $(\mathrm{P}<0.001)[20]$.On the same hand, Atta et al. [14], reported a higher mean values for HCC cases than cirrhotic cases. Also Hsia et al. [21], reported that mean value of AFP was higher in $\mathrm{HCC}$ group than hepatitis $\mathrm{C}$ and control group, and Baig et al. [22], found that, mean AFP levels in HCC patients were $421 \mathrm{ng} / \mathrm{ml}$, and concluded that, AFP is a significant marker and an indicator for Hepatocellular carcinoma.

Mean platelet volume (MPV) is a parameter of routine blood count which was actively investigated in many liver diseases.

In this work, HCC group and cirrhotic group show higher levels of MPV compared to chronic HCV group and control group $(10.94 \pm 1.88 \mathrm{fL}$ in HCC group vs $10.74 \pm 1.21 \mathrm{fL}$ in cirrhotic group vs $9.13 \pm 1.74 \mathrm{fL}$ in $\mathrm{HCV}$ group vs $8.07 \pm 0.86 \mathrm{fL}$ in control group) with highly statistically significant difference $(\mathrm{P}<0.001)$ but there was no statistically significant difference between HCC group and cirrhotic group ( $\mathrm{p}$ value $=0.49)$. These results matched with those reported by Metwaly et al. [20], who found that MPV were higher in patients with HCC and in patients with liver cirrhosis when compared with controls and patients with $\mathrm{CHC}(\mathrm{P}<0.001)$. However, no significant differences were found between patients with LC and those with HCC $(\mathrm{P}=0.94)$. Also, Kurt et al. [23], reported that mean level of MPV was higher in HCC group than cirrhotic and $\mathrm{CHC}$ groups $(9.7 \mathrm{fL}$ in HCC group, 9.1 fL in cirrhotic group and $8.6 \mathrm{fL}$ in $\mathrm{CHC}$ group).
In our study, MPV/PC ratio was higher in cirrhotic group $\left(0.19 \pm 0.09 \mathrm{fL} / 10^{9} / \mathrm{L}\right)$ than $\mathrm{HCC}$ group $\left(0.14 \pm 0.08 \mathrm{fL} / 10^{9} / \mathrm{L}\right)$, chronic hepatitis group $\left(0.05 \pm 0.02 \mathrm{fL} / 10^{9} / \mathrm{L}\right)$ and control group $\left(0.03 \pm 0.01 \mathrm{fL} / 10^{9} / \mathrm{L}\right)$ with statistically significant difference $(\mathrm{P}<0.001)$ between all the studied groups but no significant differences were found between cirrhotic group and HCC group $(p=0.06)$. Similarly, Metwaly et al. [20], found that MPV/PC ratio was higher in patients with HCC and in patients with liver cirrhosis $(\mathrm{P}<0.001)$ when compared with controls and patients with CHC. Although the MPV /PC ratio was higher in cirrhotic group $\left(1.48 \pm 0.79 \mathrm{fl} 10^{-4}\right.$ $\left.\mu 1^{-1}\right)$ than HCC group $\left(1.33 \pm 0.7 \mathrm{fl} 10^{-4} \mu \mathrm{l}^{-1}\right)$ but did not reach significant level $(\mathrm{P}=0.69)$.Also, these results were consistent with those of Cho et al. [24], who reported that MPV/PC ratio was higher in HCC group than control group with statistically significant difference $(\mathrm{P}<0.001)$ $\left(0.058 \mathrm{fL} / 10^{9} / \mathrm{L}\right.$ in $\mathrm{HCC}$ group vs $0.033 \pm 0.01$ $\mathrm{fL} / 10^{9} / \mathrm{L}$ in control group).

In present study, there was insignificant positive correlation between MPV and AFP in the HCC group ( $\mathrm{r}=0.11, \mathrm{p}=0.57)$ that is in line with Kurt et al. [23], who mentioned that there was no correlation between MPV and AFP ( $r=0.242)$. Also, the present study revealed that there was insignificant negative correlation between MPV/PC ratio and AFP in HCC group which was in agreement with Cho et al. [24], who concluded also that there was no correlation between MPV/PC ratio and AFP.

Analysis of MPV by ROC curve in the present work, showed that at cut off level $=10.7 \mathrm{fl}$, MPV had high sensitivity (73.33\%), specificity (70\%), and AUC was 0.7 in diagnosis of HCC. These results are concided with the results of Metwaly et al. [20], who demonstrated that at cut off level $=10.1 \mathrm{fl}$, MPV showed a sensitivity $(70 \%)$, and specificity $(57.3 \%)$ and AUC was 0.67 , while Kurt et al. [23], reported that at cut off level $=$ $9.2 \mathrm{fl}$, MPV showed more low sensitivity (68.3\%), and specificity (62.1\%) and AUC was 0.67 . This difference may be partially attributed to the different sample size and different studied population as Kurt et al. [23], conducted his study on 230 Turkish patients while our study was conducted only on 120 Egyptian patients.

In the present study, analysis of MPV/PC ratio by ROC curve showed that at cut off level $=0.11$ fl $/ 10^{9} / \mathrm{L}, \mathrm{MPV} / \mathrm{PC}$ ratio had a sensitivity $(76.67 \%)$, specificity $(56.67 \%)$ in diagnosis of $\mathrm{HCC}$ with 
AUC was 0.63. These results are close to the results of Metwaly et al. [20], who demonstrated that at cut off level $=0.82, \mathrm{MPV} / \mathrm{PC}$ ratio showed a sensitivity (79.6\%), and specificity $(72.7 \%)$ and AUC was 0.777. While Cho et al. [24], who reported that The AUC for MPV/PC ratio was 0.884 with high sensitivity (74.5\%), and specificity $(96.5 \%)$ at the criterion $=0.0491$ (a vertical arrow). This difference between may be attributed to the different sample size and different studied population as Cho et al. [24], conducted his study on 411 Korean patients while our study was conducted on 120 Egyptian patients.

Regarding AFP, by ROC curve analysis in the present study, at cut off level $=62.3 \mathrm{ng} / \mathrm{dl}$, it showed a higher sensitivity (90.0\%), and higher specificity (98.3\%) than MPV and MPV/PC ratio with AUC was 0.97 in diagnosis of HCC. This result was agreed with Metwaly et al. [20], who conducted his study on 200 Egyptian patients and found that at cut of level $=16.9 \mathrm{ng} / \mathrm{dl}$ had high sensitivity $(81 \%)$ and specificity $(82 \%)$ in detection of HCC than MPV and MPV/PC ratio with AUC was 0.88 , so he concluded that that MPV and MPV/PC ratio are less sensitive and specific than AFP as markers for HCC. Therefore, they may be used only in association with other markers like AFP to improve sensitivity of tumor detection. But this result disagreed with Kurt et al. [23], and Cho et al. [24], who reported higher sensitivity and specificity for MPV and MPV/PC ratio than AFP in their studies. This difference may be attributed to the different sample size, different studied population or difference in HCV genotype.

\section{REFERENCES}

1- Ferlay J, Soerjomataram I, Dikshit R, Eser S, Mathers C, Rebelo M. Cancer incidence and mortality worldwide: sources, methods and major patterns in GLOBOCAN 2012.Int J Cancer. 2015 Mar 1;136 (5):E359-86.

2- Chen CJ, Yang $\mathrm{H}$ and Iloeje UH. Hepatitis B virus DNA levels and outcomes in chronic hepatitis B. Hepatology 2009; 49: 72-84.

3- Lopez JB. Recent development in the first detection of hepatocellular carcinoma. Clin. Biochem. Rev 2005; 26: 65-79.

4- Purnak T, Efe C, BeyazitY, Ozaslan E, Astan R, Milanlioglu A et al.Recent insights into the relationship between inflammatory liver diseases and atherosclerosis. J Investig Med 2011; (59): 904-911.

5- Karaman H, Karakukcu C, Karaman A, Kayman $\mathrm{T}$, Yalsin S, Tasdemir E et al. Mean platelet volume as a fibrosis marker in patients with chronic hepatitis C. Turk J Med Sci 2013 ;(43): 39-45.

6- Martinez MG, Caamano AI, Torres EP ,Gordillo FS, Gutierrez XR and De la Tiegra FH. Mean platelet volume as a novel predictor of systemic inflammatory response in cirrhotic patients with culture-negative neutrocytic ascites. World $J$ Hepatol 2015; 7(7): 1001-1006.

7- Bessman JD, Gilmer PR and Gardner FH.Use of mean platelet volume improves detection of platelet disorders. Blood Cells 1985; (11):127135 .

8- Karnad A and Poskitt TR. The automated complete blood cell count. Use of the red blood cell volume distribution width and mean platelet volume in evaluating anemia and thrombocytopenia. Arch Intern Med 1985; (145): 1270-1272.

9- Howarth S, Marshall LR, Barr AL, Evans S, Pontre $\mathrm{M}$ and Ryan $\mathrm{N}$.Platelet indices during normal pregnancy and pre-eclampsia. $\mathrm{Br} J$ Biomed Sci 1999; (56):20-22.

10- Yilmaz MB, Saricam E, Biyikoglu SF, Guray Y, Guray U, Sasmaz H .Mean platelet volume and exercise stress test. J Thromb Thrombolysis 2004; (17):115-120.

11- Llovet JM, Ducreux M, Lencioni R, Di Bisceglie AM, Galle PR, Dufour JF, et al. EASL-EORTC Clinical Practice Guidelines: Management of hepatocellular carcinoma. J. Hepatol 2012 ; (56): 908-943.

12- Cheng A, Kang Y, Chen Z, Tsao CJ, Qin S, Kim JS et al. Randomized phase III trial of sorafenib versus placebo in Asian patients with advanced hepatocellular carcinoma: a phase III randomised, double-blind, placebo-controlled trial. Lancet Oncol. 2009; 10(1):25-34.

13- Sherlock S, Dooley J, Lok AS, Burroughs AK and Heathcote EJ. Diseases of the liver and biliary system. 12th ed., Blackwell S.C., Oxford, London, Eninburgh, 2011, Chapter (35), page 681-703.

14- Atta MM, El-Masry SA, Abdel-Hameed M, Baiomy HA and Ramadan N. Valueofserumantip53 antibodies as prognostic factorin Egyptian patients with hepatocellular carcinoma. Clinical Biochemistry 2008 ;(41): 1131-1139.

15- El-Shahat A, Swelim M, Mohamed A and AbdelWahhab M .Correlation study between aflatoxin M1 and hepatitis C virus in Egyptian patients with chronic liver disease. World Journal of Medical Sciences 2012; 7 (4): 224-231.

16- Abdel-Wahab M, El-Ghawalby N, Mostafa M, Sultan A, El-Sadany M, Fathy $O$ et al. Epidemiology of hepatocellular carcinoma in lower Egypt, Mansoura Gastroenterology Center. Hepatogastroenterology 2007; (54):157-162.

17- El-Zayadi AR, Badran HM, Barakat EM, Attia Mel-D, Shawky S, Mohamed MK et al. 
Hepatocellular carcinoma in Egypt: a single center study over a decade World J Gastroenterol 2005; (11): 5193- 5198 .

18- Ozer B, SerinE, Yilmaz U, Gimirdili Y, Saygili $\mathrm{OB}$, Kayasel $\mathrm{F}$ et al. Clinicopathologic features and risk factors for hepatocellular carcinoma: results from a single center in southern Turkey. Turk. J. Gastroenerol 2003; 14(2): 85-90.

19- Ryder SD .Guidelines for the diagnosis and treatment of hepatocellular carcinoma (HCC) in adults. Gut 2003; 52 (III): 1-8.

20- Metwaly K, Abdel Sameea E, EI-Azab G, Assem M,Abbas M, Zakaria $\mathrm{T}$ et al. Mean platelet volume and mean platelet volume/ platelet count ratio asmarkers for hepatocellular carcinoma in patients with chronic hepatitis C virus related cirrhosis. J. Cancer Res. Exp. Oncol 2016; 8(4): 33-40.

21- Hsia CY, Huo Tl, Chiang SY, Lu MF, Sun CL,
$\mathrm{Wu}$ JC etal. Evaluation of interleukin-6, interleukin-10 and humanhepatocyte growth factor a stumor markers for hepatocellular carcinoma. EJSO 2007; 33(2):208-212.

22- Baig JA, Alam JM, Mahmood SR, Baig M, Shaheen R, Sultana I et al. Hepatocellular carcinoma (HCC) and diagnostic significance of A-fetoprotein (AFP). J Ayub Med Coli Abbottabad 2009; 21(1):72-75.

23- Kurt M, Onal I, Sayilir A, Beyazit Y ,Oztas E, Kekilli $M$ et al. The Role of Mean Platelet Volume in the Diagnosis of Hepatocellular Carcinoma in Patients with Chronic Liver Disease. Hepato-Gastroenterology 2012; 59(117): 1580-1582.

24- Cho SY, Yang JJ, You E, Kim BH, Shim J, Lee $\mathrm{HJ}$ et al .Mean Platelet Volume/Platelet Count in Hepatocellular carcinoma. Platelet 2013; (5):375377. 